\title{
Multirate Kalman Filtering Approach for Optimal Two-Dimensional Signal Reconstruction from Noisy Subband Systems
}

\author{
J.Q. $\mathrm{Ni}^{*}$ \\ K.L. Ho ${ }^{*} \quad$ K.W. Tse ${ }^{*}$ and J.S. Ni ${ }^{* *}$
${ }^{*}$ Department of Electrical and Electronic Engineering \\ The University of Hong Kong, Pokfulam Road, Hong Kong \\ jqni@eee.hku.hk
}

${ }^{* *}$ Wuhan University of $H_{i}$ draulic and Electric Engineering, Wuhan, P. R. China

\begin{abstract}
Conventional synthesis filters in subband systems lose their optimality when additive noise due, for example, to signal quantization, disturbs the subband components. The multichannel representation of subband signal is combined with the statistical model of input signal to derive the multirate state-space model for filter bank system with additive noises. Thus the signal reconstruction problem in subband system can be formulated as the process of optimal state estimation in the equivalent multirate state-space model. With the input signal embedded in the state vector, the multirate Kalman filtering provides the minimum-variance reconstruction of input signal. Using the powerful Kronecker product notation, the results and derivations can then be extended to the 2-D cases. Incorporated with the vector dynamical model, the 2-D multirate state-space model for 2-D Kalman filtering is developed. Computer simulation with the proposed 2-D multirate Kalman filter gives favorable results.
\end{abstract}

\section{Introduction}

Recently multirate signal processing has become the domain of extensive research. The concept of multirate signal processing is to decompose the original signal into complementary frequency bands and then process them separately in each subband. There have been tremendous progress on the design of decimation and interpolation filters or analysis/synthesis filter banks that allow perfect reconstruction[5]. The conventional approach for $\mathrm{PR}$ filter bank systems are based on the assumption that the subband components are free of noise. However, in practical application with filter bank systems, the subband components are always contaminated by noises due to the effect of quantization, round off and other corruption, therefore, the perfect reconstruction is no longer possible.

The goal of the paper is to improve the applicability of filter bank systems by proposing the multirate Kalman synthesis filter to replace the conventional synthesis filters, with emphasis on 2-D cases. With the input signal embedded in the state vector, the multichannel representation of subband signals is combined with the statistical model of input signal to derive the multirate state-space model for the filter bank systems and the subband noises are assumed to be additive ones in this model. On the basis of this model, the multirate Kalman filter can be constructed to provide the minimumvariance estimation of the input signal based on observations of noisy subband components. The concept of multirate Kalman synthesis filtering is first given in [3]. Our works differ from those of [3] in that the philosophy to derive the state-space model is different and the multirate state-space model suitable for 2-D Kalman filtering is developed.

\section{Filter bank Systems and Problem Formulation}

\section{II.l Filter bank systems}

$$
\text { Let }\left\{\mathbf{H}_{k}(z), \mathbf{G}_{k}(z): k=0,1, \cdots, M-1\right\} \text { denotes the } M
$$
band filter bank systems. The bank of filters $\left\{\boldsymbol{H}_{k}(z)\right\}$ constitutes the analysis filters. Each filter output is downsampled and transmitted to the receiver, where they are up-sampled and fed into the bank of synthesis filters $\left\{\mathbf{G}_{k}(z)\right\}$ for signal reconstruction.

\section{II.2 Multichannel representation of subband signals}

Without any loss of generality, we shall consider only the $M=2$, i.e., two band case in the interest of convenient description, the derivations and results can be easily generalized to $M>2$ case at the cost of notational compiexity. Let $\left\{h_{i}, g_{i}: i=0.1\right\}$ denotes the impulse response of 2 -band analysis/synthesis filter bank, we have the equivalent multichannel representation of subband signals $y_{i}(n), i=0,1$

$$
\begin{array}{cc}
\mathbf{y}(\mathrm{n}) & =\sum_{k=-\lambda}^{\Lambda} \mathbf{H}(k) \mathbf{f}(n-k) \\
\text { where } \quad \mathbf{f}(n) & =\left[\begin{array}{l}
f_{0}(n) \\
f_{1}(n)
\end{array}\right]=\left[\begin{array}{c}
f(2 n) \\
f(2 n+1)
\end{array}\right], \quad \mathbf{y}(n)=\left[\begin{array}{l}
y_{0}(n) \\
y_{1}(n)
\end{array}\right]
\end{array}
$$

and $\mathbf{H}(k)$ is the multichannel impulse response matrix of the form

$$
\begin{gathered}
\mathbf{H}(k)=\left[\begin{array}{ll}
h_{00}(k) & h_{01}(k) \\
h_{10}(k) & h_{11}(k)
\end{array}\right] \\
h_{i 0}(k)=h_{i}(2 k) \text { and } h_{i 1}(k)=h_{i}(2 k-1) i=0,1
\end{gathered}
$$

The $h_{i j}(n)$ and $f_{i}(n)$ above are the so-called polyphase 
components of $h_{i}(n)$ and $f(n)$ respectively.

If additive noisy corruption are included in the subband components, the received subband signal $\mathbf{r}(n)$ can be expressed as follows:

$$
\mathbf{r}(n)=\mathbf{y}(n)+\mathbf{v}(n)
$$

$\mathbf{v}(n)=\left[\begin{array}{ll}v_{0}(n) & v_{1}(n)\end{array}\right]^{\tau}$ in (2-3) is the additive-noise disturbance vector.

\section{Derivation of Multirate State-Space Model for 1-D Signal}

\section{III.1 The Basic Signal Model}

Recall the multichannel representation of subband signal (2-1). Let $p=\lambda+\Lambda$, where $\lambda$ and $\Lambda$ are chosen such that $h_{i}(-2 \lambda-1)=0$ and $h_{i}(2 \Lambda+1)=0 ; i=0,1$. By substituting $k=k-\lambda$,we can rewrite $(2-1)$ as a causal form

$$
\mathbf{y}(\mathbf{n})=\sum_{k=0}^{P} \mathbf{H}(k-\lambda) \mathbf{f}(n+\lambda-k)
$$

(3-1) can be equivalently described by a state-space model $(\mathbf{A}, \mathbf{B}, \mathbf{C}, \mathbf{D})$. If we denote the state vector as $\mathbf{x}(n)=\left[\mathbf{f}(n+\lambda-1)^{\tau} \mathbf{f}(n+\lambda-2)^{\tau} \cdots \mathbf{f}(n-\Lambda)^{\tau}\right]^{\tau}, \quad$ we then have

$$
\mathbf{x}(n+1)=\mathbf{A} \mathbf{x}(n)+\mathbf{B f}(n+\lambda)
$$

We further denote $H_{i}(k)=\left[h_{i 0}(k) h_{i 1}(k)\right]$ and notice that $h_{i}(-2 \lambda-1)=0$, the subband output can be written as

$$
\begin{array}{r}
\left.\mathbf{y}(n)=\sum_{k=1}^{p}\left[\begin{array}{l}
H_{0}(k-\lambda) \\
H_{1}(k-\lambda)
\end{array}\right] f(n+\lambda-k)+\left[\begin{array}{cc}
h_{0}(-2 \lambda) & h_{0}(-2 \lambda-1) \\
h_{5}(-2 \lambda) & h_{1}(-2 \lambda-1)
\end{array}\right] \begin{array}{c}
f[2(n+\lambda)] \\
f[2(n+\lambda)+1]
\end{array}\right] \\
=\mathbf{C x}(n)+\mathbf{D} f[2(n+\lambda)]
\end{array}
$$

Considering the effect of noisy disturbance vector $v(n)$, we can formulate the problem as the form of statespace model

$$
\begin{aligned}
& \mathbf{x}(n+1)=\mathbf{A x}(n)+\mathbf{B f}(n+\lambda) \quad \mathbf{x}(-1)=\mathbf{x}_{-1} \\
& \mathbf{r}(n)=\mathbf{C x}(n)+\mathbf{D} f[2(n+\lambda)]+\mathbf{v}(n)
\end{aligned}
$$

\section{2 The Statistical Model}

The state space model describing the statistical characteristic of input signal should have the form[1]

$$
\begin{aligned}
\mathbf{z}^{\prime}(k+1) & =\Phi \mathbf{z}^{\prime}(k)+\mathbf{G} u(k) \quad \mathbf{z}^{\prime}(-1)=\mathbf{z}_{-1}^{\prime} \\
f(k) & =\mathbf{H z} \mathbf{z}^{\prime}(k)
\end{aligned}
$$

where the $z^{\prime}(k)$ and $u(k)$ are the state vector and driving source, respectively.

Examining the state-space models described by (3-3) and (3-4) reveals that the two systems evolve in different time scale. After some algebra and let $\mathbf{z}(n)=\mathbf{z}^{\prime}[2(n+\lambda)]$, we have the equivalent block generation model (3-5) of the form:

$$
\begin{aligned}
& \mathbf{z}(n+1)=\Phi^{2} \mathbf{z}(n)+\mathbf{G}_{2} \mathbf{u}_{2}(n) \\
& \mathbf{f}(n+\lambda)=\mathrm{H}_{2} \mathbf{z}(n)+\mathbf{Q}_{2} \mathbf{u}_{2}(n) \\
& f[2(n+\lambda)]=\mathrm{Hz}(n)
\end{aligned}
$$

where $\quad \mathbf{u}_{2}(n)=\left[\begin{array}{c}u[2(n+\lambda)] \\ u[2(n+\lambda)+1]\end{array}\right], \quad \mathrm{H}_{2}=\left[\begin{array}{c}\mathrm{H} \\ \mathrm{H} \Phi\end{array}\right]$, $\mathbf{G}_{2}=\left[\begin{array}{ll}\Phi \mathbf{G} & \mathbf{G}\end{array}\right]$ and $\mathbf{Q}_{2}=\left[\begin{array}{cc}0 & 0 \\ \mathbf{H G} & 0\end{array}\right]$

\section{III.3 The Multirate State-Space Model}

The basic model (3-3) can be augmented with the statistical model (3-5) to yield the final combined multirate state-space model.

$$
\begin{aligned}
\mathbf{w}(n+1) & =\overline{\mathbf{A}} \mathbf{w}(n)+\overline{\mathbf{B}} \mathbf{u}_{2}(n) \quad \hat{\mathbf{w}}(-1)=\hat{\mathbf{w}}_{-1} \\
\mathbf{r}(n) & =\overline{\mathbf{C}} \mathbf{w}(n)+\mathbf{v}(n)
\end{aligned}
$$

where $\mathbf{w}(n)=\left[\mathbf{x}(n)^{\tau} \mathbf{z}(n)^{\tau}\right]^{\tau}$, is the state vector of system model (3-6). The parameter matrices $\overline{\mathbf{A}}, \overline{\mathbf{B}}$ and $\overline{\mathbf{C}}$ are determined as follows

$\overrightarrow{\mathbf{A}}=\left[\begin{array}{cc}\mathbf{A} & \mathbf{B H}_{2} \\ \mathbf{0} & \Phi^{2}\end{array}\right], \overline{\mathbf{B}}=\left[\begin{array}{c}\mathbf{B} \mathbf{Q}_{2} \\ \mathbf{G}_{2}\end{array}\right]$ and $\overline{\mathbf{C}}=\left[\begin{array}{ll}\mathbf{C} & \mathbf{D H}\end{array}\right]$

\section{Derivation of Multirate State-Space Model for 2-D Signal}

IV.1 The Basic Signal Model

When implementing the 2-D filter banks, the dominant approach is to apply two 1-D filter banks separately. Let $\left\{h_{i}^{c}, g_{i}^{c}: i=0,1\right\}$ and $\left\{h_{i}^{r}, g_{i}^{r}: i=0,1\right\}$ denote two 1-D filter banks for column- and row-wise operations, respectively. The original 2-D signal $f(m, n)$ can then be decomposed into four subband components $y_{i j}(m, n) i, j=0,1$ through the 2-D separate analysis banks. We have the 2-D multichannel representation of the form

$\mathbf{y}(m, n)=\sum_{l=-\hat{\lambda}^{r}}^{k^{c}} \sum_{k=-\hat{\lambda}^{\prime}}^{\lambda^{\prime}} \mathbf{H}^{c}(l) \mathbf{F}(m-l, n-k) \mathbf{H}^{r}(k)$

where $\mathbf{F}(m, n)=\left[\begin{array}{cc}f(2 m, 2 n) & f(2 m, 2 n+1) \\ f(2 m+1,2 n) & f(2 m+1,2 n+1)\end{array}\right]$ and

$\mathbf{H}^{c}(k)=\left[\begin{array}{ll}h_{00}^{c}(k) & h_{01}^{c}(k) \\ h_{10}^{c}(k) & h_{11}^{c}(k)\end{array}\right], \quad \mathbf{H}^{r}(k)=\left[\begin{array}{ll}h_{00}^{r}(k) & h_{01}^{r}(k) \\ h_{10}^{r}(k) & h_{11}^{r}(k)\end{array}\right]$

If we further define

$\overline{\mathbf{F}}(m, n)=\left[\begin{array}{llll}\mathbf{F}\left(m-\Lambda^{c}, n\right) & \mathbf{F}\left(m-\Lambda^{c}+1, n\right) & \cdots & \mathbf{F}\left(m+\lambda^{c}, n\right)\end{array}\right]^{\tau}$ and after some algebra (4-1) can be expressed as the more compact form

$\mathbf{y}(m, n)=\sum_{k=0}^{p^{r}} \overline{\mathbf{H}}^{c} \overline{\mathbf{F}}\left(m, n+\lambda^{r}-k\right) \mathbf{H}^{r}\left(k-\lambda^{r}\right)^{r}$

where $\overline{\mathbf{H}}^{c}=\left[\begin{array}{llll}\mathbf{H}^{c}\left(\Lambda^{c}\right) & \mathbf{H}^{c}\left(\Lambda^{c}-1\right) & \cdots & \mathbf{H}^{c}\left(-\lambda^{c}\right)\end{array}\right]$.

For the sake of derivation of 2-D multirate state-space model, we express ( 4-2) in a more convenient form with the powerful Kronecker product notation, namely,

$\tilde{\mathbf{y}}(m, n)=\sum_{k=0}^{p_{*}}\left(\mathbf{H}^{r}\left(k-\lambda^{r}\right) \otimes \overline{\mathbf{H}}^{r}\right) \tilde{\overline{\mathbf{F}}}\left(m, n+\lambda^{r}-k\right)=\sum_{k=0}^{p_{k}} \mathbf{C}_{k} \tilde{\overline{\mathbf{F}}}\left(m, n+\lambda^{r}-k\right)$ 
where

$\tilde{\mathbf{y}}(m, n)=\left[\begin{array}{llll}y_{(0)}(m, n) & y_{10}(m, n) & y_{01}(m, n) & y_{11}(m, n)\end{array}\right]^{\tau}$

and $\tilde{\overline{\mathbf{F}}}\left(m, n+\lambda^{r}\right)=$

$\left[f\left[2\left(m-\Lambda^{c}\right), 2\left(n+\lambda^{r}\right)\right] \cdots f\left[2\left(m+\lambda^{c}\right)+1,2\left(n+\lambda^{r}\right)\right]\right.$

$\left.f\left[2\left(m-\Lambda^{c}\right), 2\left(n+\lambda^{r}\right)+1\right] \cdots f\left[2\left(m+\lambda^{c}\right)+1,2\left(n+\lambda^{r}\right)+1\right]\right]^{r}$

The column vector $\tilde{\overline{\mathbf{F}}}\left(m, n+\lambda^{r}\right)$ happens to be constituted by two adjacent column vectors $\mathbf{f}\left[m, 2\left(n+\lambda^{r}\right)\right]$ and $\mathbf{f}\left[m, 2\left(n+\lambda^{r}\right)+1\right]$. As in 1-D case, we define state vector as

$\mathbf{x}(m, n)=$

$\left[\begin{array}{llll}\tilde{\overline{\mathbf{F}}}\left(m, n+\lambda^{r}-1\right)^{\tau} & \tilde{\overline{\mathbf{F}}}\left(m, n+\lambda^{r}-2\right)^{\tau} & \ldots & \widetilde{\overline{\mathbf{F}}}\left(m, n-\Lambda^{r}\right)^{\tau}\end{array}\right]^{\tau}$

, and consider the effect of noisy disturbance

$\widetilde{\mathbf{r}}(m, n)=\tilde{\mathbf{y}}(m, n)+\tilde{\mathbf{v}}(m, n)$, we then have

$\mathbf{x}(m, n+1)=\mathbf{A} \mathbf{x}(m, n)+\mathbf{B} \tilde{\overline{\mathbf{F}}}\left(m, n+\lambda^{r}\right) \quad \hat{\mathbf{x}}(m,-1)=\hat{\mathbf{x}}_{m}$

$\tilde{\mathbf{r}}(m, n)=\mathbf{C x}(m, n)+\mathbf{D f}\left[m, 2\left(n+\lambda^{r}\right)\right]+\widetilde{\mathbf{v}}(m, n)$

\section{IV.2 The Vector Dynamical Model}

Consider the wide-sense stationary random field $f(t, n)$, for large portion of nature images, their autocorrelation functions $R(t, n)$ are well characterized by (4-5) [2]

$$
R(t, n)=\alpha \exp \left(-\mu_{h}|t|-\mu_{v}|n|\right)
$$

On the basis of (4-5) a vector model can be developed [2], and have the form in our configuration:

$$
\begin{aligned}
& \mathbf{z}(m, n+1)=\Phi^{2} \mathbf{z}(m, n)+\mathbf{G}_{2} \mathbf{u}_{2}(m, n) \\
& \tilde{\overline{\mathbf{F}}}\left(m, n+\lambda^{r}\right)=\mathbf{H}_{2} \mathbf{z}(m, n)+\mathbf{Q}_{2} \mathbf{u}_{2}(m, n) \\
& \mathbf{f}\left[m, 2\left(n+\lambda^{r}\right)\right]=\mathbf{H z}(m, n)
\end{aligned}
$$

where

$$
\mathbf{u}_{2}(m, n)=\left[\begin{array}{c}
\mathbf{u}\left[m, 2\left(n+\lambda^{r}\right)\right] \\
\mathbf{u}\left[m, 2\left(n+\lambda^{r}\right)+1\right]
\end{array}\right],
$$

$\mathbf{z}(\mathrm{m}, \mathrm{k})$ and $\mathbf{u}_{2}(m, k)$ are the $4\left(p_{c}+1\right)$ - dimensional state vector and driving white noise vector, respectively.

\section{IV.3 The 2-D Multirate State-Space Model}

The 2-D multirate state-space model with consideration of the statistics of object plane and noisy disturbances in subbands can be constructed by combining the basic model (4-4) with the object plane model (4-6) to yield a augmented multirate state-space model (4-7).

$$
\begin{aligned}
\mathbf{w}(m, n+1) & =\overline{\mathbf{A}} \mathbf{w}(m, n)+\overline{\mathbf{B}} \mathbf{u}_{2}(m, n) \quad \hat{\mathbf{w}}(m,-1)=\hat{\mathbf{w}}_{m} \\
\overline{\mathbf{r}}(m, n) & =\overline{\mathbf{C}} \mathbf{w}(m, n)+\widetilde{\mathbf{v}}(m, n)
\end{aligned}
$$

The vector $\mathbf{w}(m, n)=\left[\begin{array}{ll}\mathbf{x}(m, n)^{\tau} & \mathbf{z}(m, n)^{\tau}\end{array}\right]^{\tau}$ is the state vector of system (4-7). The Kalman synthesis filter equations for 2-D subband system are given below for the sake of completeness

$\hat{\mathbf{w}}(m, n)=[\mathbf{I}-\mathbf{K}(m, n) \overline{\mathbf{C}}] \overline{\mathbf{A}} \hat{\mathbf{w}}(m, n-\mathbf{1})+\mathbf{K}(m, n) \overrightarrow{\mathbf{r}}(m, n)$

$\mathbf{K}(m, n)=\mathbf{P}(m, n) \overline{\mathbf{C}}^{r}\left[\overline{\mathbf{C}} \mathbf{P}(m, n) \overline{\mathbf{C}}^{t}+\mathbf{R}(m, n)\right]^{-1}$

$\mathbf{P}(m, n+\mathbf{l})=\overline{\mathbf{A}}[\mathbf{I}-\mathbf{K}(m, n) \overline{\mathbf{C}}] \mathbf{P}(m, n) \overline{\mathbf{A}}^{\tau}+\overline{\mathbf{B}} \Omega(m, n) \overline{\mathbf{B}}^{\tau}$ with $\hat{\mathbf{w}}(m,-1)=\hat{\mathbf{w}}_{m}, \mathbf{P}(m,-11-1)=\mathbf{P}_{m}$ for $m=0,1, \cdots$. $\mathbf{R}$ and $\Omega$ are the covariance matrices of $\hat{\mathbf{v}}(n)$ and $\mathbf{u}_{2}(n)$, respectively.

\section{Numerical Results and Discussions}

Computer simulation is carried out to show the feasibility and effectiveness of the proposed 2-D Kalman filtering for optimal 2-D signal reconstruction from noisy subband systems. We compare the performance of the proposed 2-D multirate Kalman filter versus the conventional PR filters for 2-D signal reconstruction under different noise levels. The simulation was implemented on the SGI/R4000 platform with Matlab.

We adopted a set of 2-band QMF PR filter bank $\{h(n)\}$ of length 8 in our simulation [4]. The $2 \times 2$ subband decomposition of test image is implemented through two 1-D separate analysis filter bank $\left\{h_{i}^{c}, h_{i}^{r}\right\}_{i=0,1}$ with $h_{i}^{c}(n)=h_{i}^{r}(n)=h_{i}(n), i=0,1$.

Two quantitative measures, $S N R_{i}^{m, n}$ and $S N R_{r}$, are defined to reflect the input noise level and reconstruction performance, respectively. We would denote $S N R_{i}^{m . n}$ as $S N R_{i}$, since we adopted the same SNR level in each subband in our simulation. The test image Hillside is of size $160 \times 160 \times 8$ ( fig. 1 (a) ). The image is converted to zero mean prior to $2 \times 2$ subband decomposition and its autocorrelation function is found to have the form of $R(t, n)=1168.3 \exp (-23.59|t|-0.22|n|)(t:$ continuous in horizontal direction ). White additive noise at different SNR levels was added to all the four subband images. Fig. 2 demonstrates the reconstruction performance comparison with both the proposed multirate Kalman filtering and the conventional PR synthesis filters under different SNR level. It is observed that the improvement in reconstruction SNR with the proposed 2-D multirate Kalman filtering is considerable. Figl.(e) shows that, even in the extremely low $S N R_{i}$ case, the main structure of the original image is still distinguishable on the reconstructed image with $2-\mathrm{D}$ multirate Kalman filtering.

\section{Conclusion}

In this paper, we combine the multichannel representation of subband signal with the statistical model of input signal to derive the multirate state-space model for noisy filter bank systems, with emphasis on 2$D$ cases. With the input signal embedded in the state vector, the issue of signal reconstruction can be formulated as the optimal state estimation with multirate Kalman filtering. Since the Kalman filtering is onedimensional in nature, the vector dynamical model is incorporated to develop the 2-D multirate state-space model suitable for 2-D multirate Kalman filtering. Computer simulation results demonstrate significant improvement in $S N R_{r}$ of reconstructed 2-D signal, under different noise levels, with the proposed 2-D multirate 
Kalman filtering approach over those with conventional synthesis filter. The nonstationarity of nature images, however, can be tackled, with adaptive Kalman filtering approach based on the image segmentation, which remains the topic for further research.

\section{References}

[1] N.E. Nahi, Estimation Theory and Applications, New York:Wiley, 1969.

[2] N.E. Nahi, "Recursive Image Enhancement-Vector Processing," IEEE Trans. Com., Vol. COM-21, pp.305311 , Apr. 1973.
[3] B. Chen, "Optimal Signal Reconstruction in Noisy Filter Bank Systems: Multirate Kalman Synthesis Filtering Approach," IEEE Trans. Signal Processing, Vol.43, pp.2496-2504, Nov., 1995.

[4] A.Delopoulos, "Optimal Filter Banks for Signal Reconstruction from Noisy Subband Components," IEEE Trans. signal processing, Vol. 44, pp212-224, Feb.,1996. [5] P.P. Vaidyanathan, "Multirate Digital Filters, Filter Banks, Polyphase Networks, and Applications: A Tutorial ,'Proc. of The IEEE, Vol.78, No.1, pp.56-93, Jan. 1990.

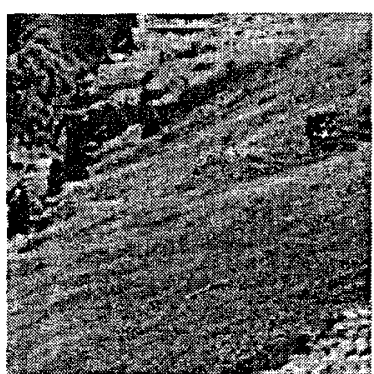

( a )

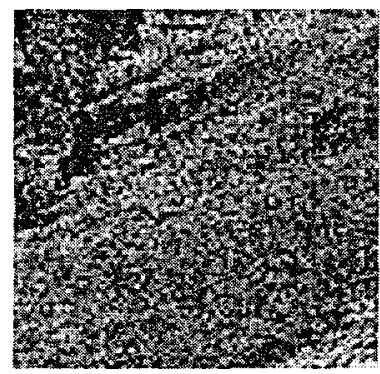

(d)

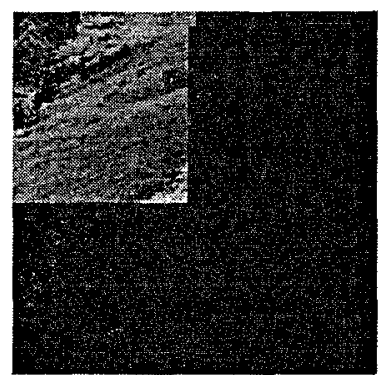

(b)

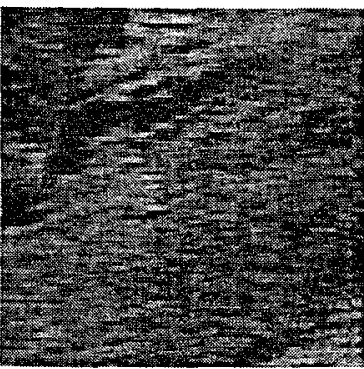

(e)

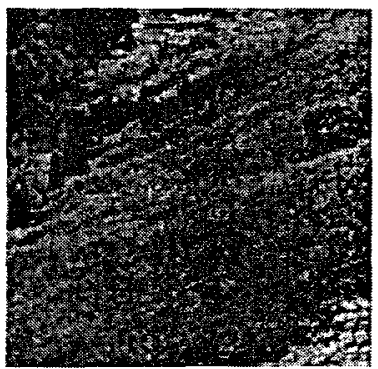

(f)

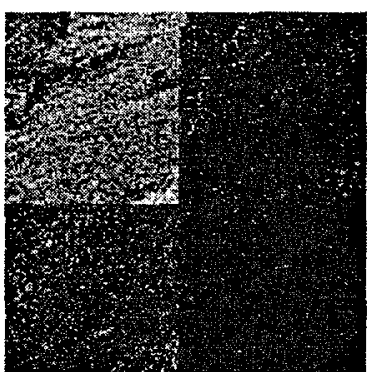

(c)

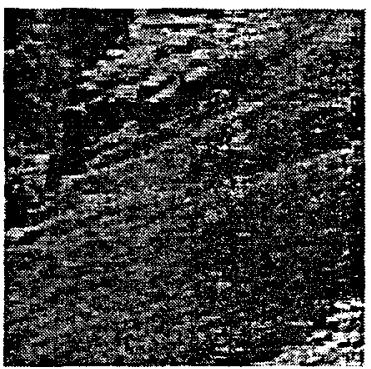

( $g$ )

Fig. 1. (a) Original image Hillside of size $160 \times 160$; (b) $2 \times 2$ subband decomposition of Hillside; (c) noise-corrupted subband image at $S N R_{i}=0 \mathrm{db}$; (d) reconstructed image with conventional PR filter banks at $S N R_{i}=0 \mathrm{db}$; (e) reconstructed image with 2-D Kalman filtering at $S N R_{i}=0 \mathrm{db}$; (f) reconstructed image with conventional PR filter banks at $S N R_{i}=8 \mathrm{db} ;(\mathrm{g})$ reconstructed image with 2-D Kalman filtering at $S N R_{i}=8 \mathrm{db}$.

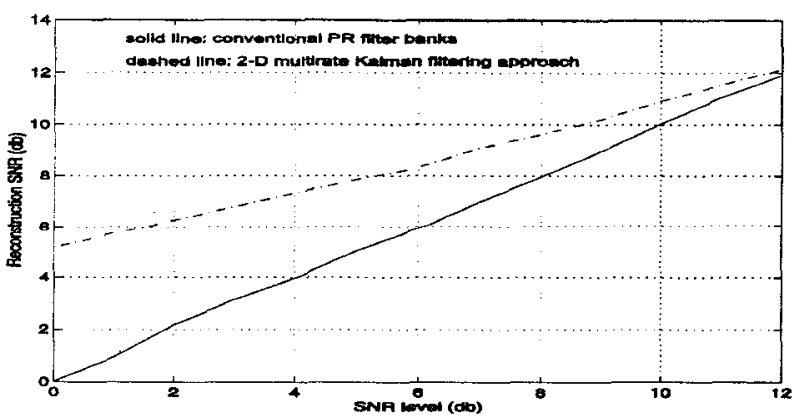

Fig. 2 Reconstruction SNR versus additive noise SNR level 